\title{
Theoretical Study of Peculiarities of Unstable Longitudinal Shear Crack Growth in Sub-Rayleigh and Supershear Regimes
}

\author{
Evgeny V. Shilko ${ }^{1,2, a)}$ and Sergey G. Psakhie ${ }^{1,3, b)}$ \\ ${ }^{1}$ Institute of Strength Physics and Materials Science SB RAS, Tomsk, 634055, Russia \\ ${ }^{2}$ National Research Tomsk State University, Tomsk, 634050, Russia \\ ${ }^{3}$ National Research Tomsk Polytechnic University, Tomsk, 634050, Russia

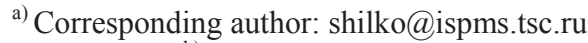 \\ b) sp@ispms.tsc.ru
}

\begin{abstract}
In the paper we present the results of the theoretical study of some fundamental aspects of mode II crack propagation in conventional sub-Rayleigh regime and transition to intersonic regime. It is shown that development of a sub-Rayleigh shear crack is determined in many respects by elastic vortex traveling ahead of the crack tip at a shear wave velocity. Formation of such a vortex helps to better understand the well-known phenomenon of acceleration of a shear crack towards the longitudinal wave velocity. Simulation results have shown that due to self-similarity of shear crack propagation the conditions of sub-Rayleigh to intersonic transition depend on dimensionless material and crack parameters. Two key dimensionless parameters are proposed.
\end{abstract}

Keywords: dynamic mode II fracture, Interface crack, sub-Rayleigh regime, supershear regime, self-similarity, computer-aided simulation, movable cellular automaton method

\section{INTRODUCTION}

The question about physically admissible velocity of unstable (dynamic) crack growth is of significance to safety engineering as well as to earthquake dynamics. In the framework of conventional fracture mechanics analytical predictions, a brittle crack cannot propagate faster than the Rayleigh wave. Nevertheless, recent researches including numerical simulations, experimental observations and the analysis of strong earthquakes have shown a possibility of faster propagation of mode II cracks at velocities comparable with longitudinal wave (P-wave) speed $V_{\mathrm{P}}$.

A considerable number of reports regarding intersonic and supersonic shear crack propagation in full-scale systems, laboratory experiments and computer-aided simulations have been published for the last four decades [1-6]. An interest to this problem started from pioneering works of Burridge [1] and Andrews [2]. Andrews first numerically studied the main features of mode II crack propagation with the use of finite element model. In particular he has shown that the rupture initially propagates at a significant fraction of the Rayleigh velocity $V_{R}$ and the stress peak appears in the crack tip at an early stage of shear crack development. This stress peak propagates ahead of the rupture front at a shear wave velocity $V_{\mathrm{S}}$ (a little bit faster than Rayleigh velocity) and gradually becomes higher. It potentially can reach yield stress and induce fracture ahead of the shear crack tip [2, 3]. This secondary rupture propagates faster than the shear wave. During the following decades a lot of results concerning supershear crack propagation regime were drawn. Different authors experimentally or numerically observed mode II crack propagation in intersonic regime in the whole range of length scales from atomic one [3] to macroscopic scale [6]. It is important that supershear rupture is observed in elastic-brittle or quasi-brittle materials, while unstable crack growth in conventional sub-Rayleigh regime is inherent to materials with various rheological characteristics.

Despite huge success in studying intersonic crack propagation, some issues related to dynamics and conditions of mode II crack acceleration to intersonic velocity are still not fully understood. The present paper is addressed to the

International Conference on Physical Mesomechanics of Multilevel Systems 2014

AIP Conf. Proc. 1623, 571-574 (2014); doi: 10.1063/1.4901500

(C) 2014 AIP Publishing LLC 978-0-7354-1260-6/ $\$ 30.00$ 
issues concerning the conditions (including influence of material properties and geometrical characteristics of cracks), which make possible the propagation of mode II crack at a velocity higher than the shear elastic wave speed.

\section{PROBLEM STATEMENT}

The study was carried out by means of movable cellular automaton (MCA) based on numerical simulation. The MCA method is a representative of particle-based numerical techniques, which treat the solid as an ensemble of bonded (or linked) interacting particles [7]. To study the considered problem the model of many-body interaction between movable cellular automata, which provide macroscopically isotropic linear-elastic response of particle ensemble, was used [7].

The numerical study was carried out with the use of the two-dimensional model sample consisting of two bonding parts [8]. Parts have the same properties and are considered as isotropic, linear elastic and high-strength. The following mechanical properties of material of the parts were used in the study: Young modulus $E=200 \mathrm{GPa}$, Poisson's ratio $v=0.3$, density $\rho=5700 \mathrm{~kg} / \mathrm{m}^{3}$. Ideal bonding of the plates was assumed. In the framework of such assumption the only interface property taken into account was interface strength. The value of interface strength $(250 \mathrm{MPa})$ was assumed to be much smaller than strength of plates themselves (2000 MPa). The interface contained section-shaped initial crack [8]. This implies the case of extremely narrow cracks (there is no gap between crack surfaces before loading). Simple shear loading was applied to the sample. This was simulated by displacing of the upper and lower surfaces of the sample in horizontal direction with very low constant velocity [8]. Plane strain state approximation was used in described 2D model.

\section{SIMULATION RESULTS AND DISCUSSION}

Simulation results have shown that the most important feature of dynamic propagation of a mode II crack is formation and development of a collective elastic rotational motion of material particles ahead of the crack tip. Figure 1(a) shows a snapshot of a typical velocity field near the right tip of dynamically growing mode II crack, shortly after unstable crack propagation beginning. The collective elastic rotational motion (hereinafter referred to as elastic vortex) ahead of the crack is clearly seen. Such a vortex forms immediately after crack starts to propagate. As the crack advances, the elastic vortex rapidly increases in size, and its velocity approaches the shear wave speed $V_{\mathrm{S}}$. At the same time mode II crack generating this vortex propagates at a lower velocity amounting to considerable fraction of the Rayleigh wave speed $V_{\mathrm{R}}$. Therefore, shortly after the start of dynamic crack growth an elastic vortex leaves the crack tip behind and then develops as a self-dependent dynamic object.

The most important feature of elastic vortex travelling ahead of propagating crack is concentration of shear stresses. The region of high shear stresses is positioned in the frontal part of the vortex and has an elliptical shape (Fig. 1(b)). The values of shear stresses in this region gradually change during the course of vortex development. They continuously increase till the moment of vortex separation from the crack and then gradually decrease as the vortex travelling independently of the crack attenuates. So, the maximum stress concentration in the vortex is reached at the moment of vortex detaching from the crack.

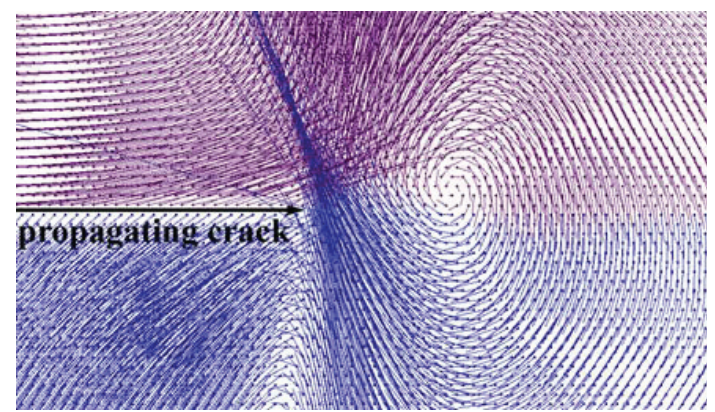

(a)

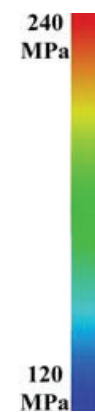

MPa

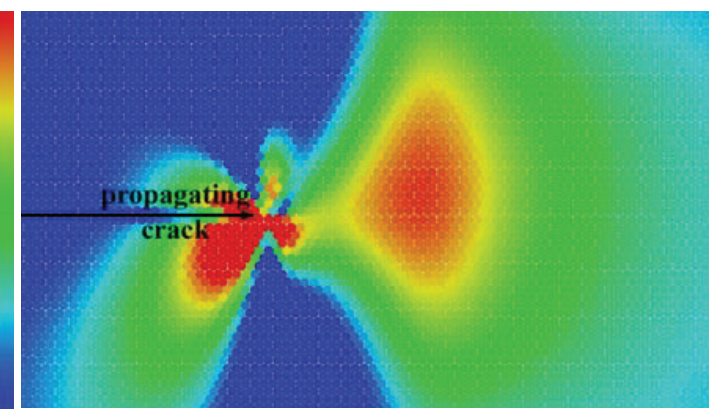

(b)

FIGURE 1. Typical field of velocities of movable cellular automata near the tip of growing shear crack (a) and corresponding distribution of equivalent stresses (b) shortly after unstable crack propagation beginning 
Described feature determines a capability of the mode II crack to accelerate towards the longitudinal wave speed $V_{\mathrm{P}}$. Such acceleration can take place if equivalent stress at the interface ahead of the crack tip (namely at the central point of the section of elliptical region of high shear stresses by interface line) reaches the value of interface strength before the vortex detaches from the crack tip. In this case a so-called daughter crack is formed at the interface ahead of the main crack and this secondary rupture propagates at a velocity faster than $V_{\mathrm{S}}[2,3,8]$.

Supershear crack propagation was observed numerically or experimentally at different scales from atomic one [3] to the scale of tectonic faults [6]. This gives grounds to suggest that the phenomenon of sub-Rayleigh to supershear crack propagation regime transition is self-similar. The assumption of self-similarity of mode II crack propagation was first used by Burridge [1]. In the present study this assumption was verified by special calculations for scaled samples. The basic model sample was scaled by multiplying the automaton and sample sizes within several orders of magnitude (the initial crack was scaled by the same factor). Simulation results have shown that scaled samples demonstrate totally similar velocity and stress/strain fields at corresponding time moments from the time of crack propagation beginning (i.e. at time moments scaled by spatial scaling factor). These results support the statement of self-similar nature of the shear crack propagation and transition to intersonic regime and indicate that criterion, which determines the possibility of overcoming the "Rayleigh speed barrier", should be determined by specific and/or dimensionless characteristics of material and initial crack. Simulation results showed that the density of elastic strain energy of distortion $W_{0}$ accumulated in the material at the moment of unstable crack growth beginning serves as such a criterion. If $W_{0}$ exceeds the critical value $W_{\text {crit, }}$, the crack can develop faster than the shear wave. Convenient characteristic of $W_{\text {crit }}$ is the shear stress at the onset of unstable crack growth $\tau_{\text {crit }}$ ( $\tau_{\text {crit }}=\sqrt{2 W_{\text {crit }} G}$, where $G$ is shear modulus of material). Hereinafter the parameter $\tau_{\text {crit }}$ will be referred to as the critical shear strength. In the paper the dependence of $\tau_{\text {crit }}$ on the material parameters and on the geometrical characteristics of the initial crack was revealed.

The most important parameters of elastic-brittle material are elastic constants $(E$ and $v)$ and density $\rho$. It is shown that dependences of $\tau_{\text {crit }}$ on these parameters can be reduced to a one-parameter dependence $\tau_{\text {crit }}\left(V_{\mathrm{R}} / V_{\mathrm{S}}\right)$ determined by the ratio of the Rayleigh wave velocity $V_{\mathrm{R}}$ to the shear wave velocity $V_{\mathrm{S}}$. Figure 2(a) shows the curve $\tau_{\text {crit }}\left(V_{\mathrm{R}} / V_{\mathrm{S}}\right)$ corresponding to the interval of Poisson's ratios from 0 to 0.45 at the same value of Young modulus $E=200 \mathrm{GPa}$ (Young modulus and density do not significantly influence the magnitude of $\tau_{\text {crit }}$ ). It is seen that increase in the ratio $V_{\mathrm{R}} / V_{\mathrm{S}}$ leads to decrease in the strength threshold by a quarter of the value.

The influence of other parameters of elastic-brittle material on critical shear strength $\tau_{\text {crit }}$ is much less pronounced. For example, hundred times increase in dynamic viscosity $\mu$ leads to two times smaller change in threshold value of shear strength $\tau_{\text {crit }}$ than variation of Poisson's ratio (Fig. 2(b)).

Supershear crack propagation is an inherent property of crack-shaped discontinuities and manifested at all spatial scales. This implies that dependence $\tau_{\text {crit }}$ on the geometry of the initial crack must be expressed in terms of dimensionless parameters, which are combinations of dimensional geometrical characteristics. We have found such a dimensionless geometrical parameter characterizing the initial crack of simple geometry (in the form of section or rectangular notch). Such kind of cracks are commonly characterized by length $X_{0}$ and effective thickness $T$.

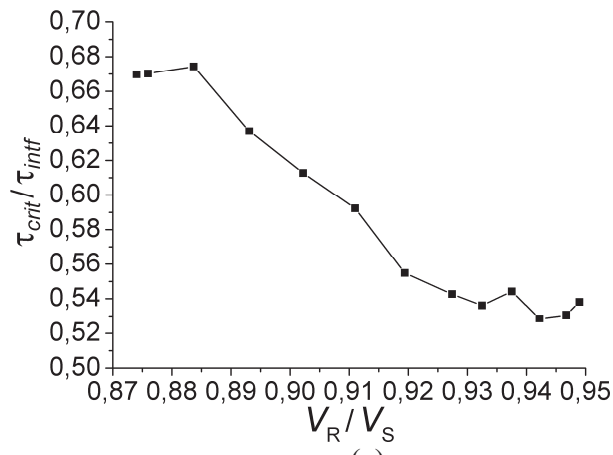

(a)

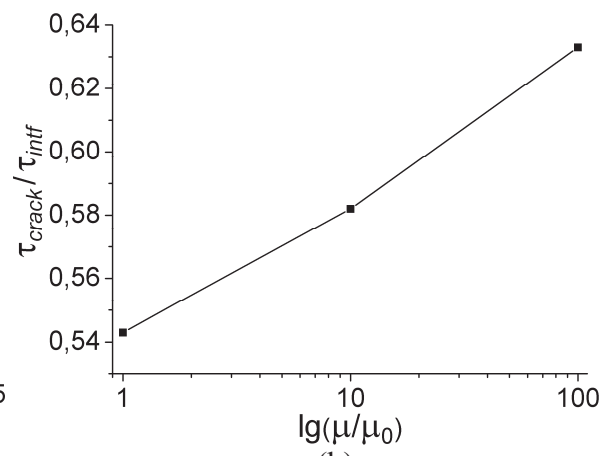

(b)

FIGURE 2. Dependences of reduced critical value of shear strength of the interface with crack $\tau_{\text {crit }}$ on the ratio of Rayleigh to shear wave speed $V_{\mathrm{R}} / V_{\mathrm{S}}$ (a) and on logarithm of reduced value of dynamic viscosity (b). Critical shear strength $\tau_{\text {crit }}$ is normalized by the shear strength of intact interface $\tau_{\text {intf }}$. Normalizing constant $\mu_{0}$ in (b) is some reference viscosity 
Simulation results showed that regardless of the specific values of $X_{0}$ and $T$ the mode II crack can propagate in supershear regime if the ratio $R=T / X_{0}$ exceeds the threshold value $R_{\text {crit }}$ (the interface with crack characterized by dimensionless parameter $R_{\text {crit }}$ has the shear strength $\tau_{\text {crit }}$ ). It follows herefrom that the effective thickness of the crack $T$ determines the maximum value of the initial crack length $L_{\text {crit }}$, at which a crack could potentially propagate at a velocity exceeding the shear elastic wave speed in the material.

\section{SUMMARY}

The study shows that the development of a shear crack is determined by an elastic vortex travelling ahead of the crack tip at a shear wave speed. The stress concentration area ahead of the crack tip revealed by different authors is a manifestation of this collective elastic rotational motion of material particles. The formation of such dynamic object makes possible the nucleation of a daughter crack and subsequent crack propagation in intersonic regime. The results of numerical study support analytically proposed statement of self-similar nature of the shear crack propagation and transition to intersonic regime and indicate the presence of dimensionless material $\left(V_{\mathrm{R}} / V_{\mathrm{S}}\right)$ and geometrical $\left(T / X_{0}\right)$ parameters governing the condition of crack propagation transition to supershear regime.

E. Shilko acknowledges financial support from the Russian Science Foundation (grant 14-19-00718). S. Psakhie wishes to thank SB RAS Program III.23.1 for Basic Research (Project No. 4) for financial support.

\section{REFERENCES}

1. R. Burridge, Geophys. J. R. Astr. Soc. 35, 439 (1973).

2. D. J. Andrews, J. Geophys. Res. 81, 5679 (1976).

3. F. F. Abraham and H. Gao, Phys. Rev. Lett. 84, 3113 (2000).

4. P. H. Geubelle and D. V. Kubair, J. Mech. Phys. Solids 49, 571 (2001).

5. E. M. Dunham, J. Geophys. Res. 112, B07302 (2007).

6. M. Mello, H. S. Bhat, A. J. Rosakis, and H. Kanamori, Tectonophysics 493, 297 (2010).

7. S. Psakhie, E. Shilko, A. Smolin, S. Astafurov, and V. Ovcharenko, Fract. Struct. Integrity 24, 26 (2013).

8. E. V. Shilko and S. G. Psakhie, Proc. Mat. Sci. 3C, 263 (2014). 